INPLASY

PROTOCOL

To cite: Ni et al. Effects of

Exercise Programs in older

Adults with Muscle Wasting: A

Systematic Review and Meta-

analysis. Inplasy protocol

202140146. doi:

10.37766/inplasy2021.4.0146

Received: 30 April 2021

Published: 30 April 2021

Corresponding author:

Heng-Hsin Tung

henghsin@ym.edu.tw

Author Affiliation:

National Yang Ming Chiao

Tung University

Support: No funding source.

Review Stage at time of this submission: Data analysis.

Conflicts of interest:

None declared.

\section{Effects of Exercise Programs in older Adults with Muscle Wasting: A Systematic Review and Meta-analysis}

$\mathrm{Ni}, \mathrm{HJ}^{1}$; Tung, $\mathrm{HH}^{2}$; Hsu, TF3; Chen, YC4; Chow, LC5; Mu, PF6; Chiou, AF7.

Review question / Objective: P:older adults with Muscle Wasting; I:Exercise; C:Usual care; O:primary outcome:muscle mass; secondary outcome:physical performance, muscle strength.

Eligibility criteria: (i) Randomized control trial; (ii) Include the diagnosis of muscle wasting disorder with an mean or median age $\geq 60$ years, including: muscle wasting, according to the EWGSOP and AWGS or Other definitions of sarcopenia, disuse atrophy, cachexia caused by cancer or heart failure, and chronic obstructive pulmonary disease (COPD). In studies where exercise interventions were delivered to patients with cancer and at risk of cachexia, domains for the syndrome including weight loss, nutritional stores and intake, and muscle mass or function were seldom reported. To this end, it was not possible to determine the proportion of patients meeting pre-cachexia or cachexia criteria; (iii) All types of exercises, resistance exercises, aerobic exercises, vibration exercises, and multi-component regime; (iv) Full text articles in Chinese and English; and (v) Outcomes of the studies included at least one of the following data results: muscle mass, muscle strength or physical function.

INPLASY registration number: This protocol was registered with the International Platform of Registered Systematic Review and Meta-Analysis Protocols (INPLASY) on 30 April 2021 and was last updated on 30 April 2021 (registration number INPLASY202140146).

\section{INTRODUCTION}

Review question / Objective: P:older adults with Muscle Wasting; I:Exercise; C:Usual care; O:primary outcome:muscle mass; secondary outcome:physical performance, muscle strength.

Rationale: Cachexia and muscle wasting are recognized as being the consequence 
of aging and variety of acute and chronic illnesses and affect millions of elderly people and patients. Both are closely associated with poor quality of life as well as significantly increased morbidity, mortality and related to increased risk of loss of physical independence. Muscle wasting represents a major risk factor for decreased muscular resistance and loss of independency in daily life activities. In the last decades, no therapies have been approved for muscle wasting or cachexia. One alternative clinical intervention that may provide the most benefits is exercise training. We performed a systematic review and meta-analysis to evaluate the available literature related to the effects of exercise interventions on muscle mass, muscle strength and physical performance in older adults with Muscle wasting.

Condition being studied: Up to date, there is no effective treatment available for the vast majority of muscle wasting. Skeletal muscle remains one of the most undermedicated organs. Therapeutic approaches include nutritional supplements, exercise, and drug treatments, but evidence remains unsatisfying. Among them, exercise has an effect on the prevention and treatment of many diseases. Most studies of older adults exercise intervention indicated that the participants achieved positive results. Although exercise is protective against sarcopenia for older adults, there are limited studies in this area. Previous systematic review regarding exercise interventions suggested muscle strength and physical performance, even muscle mass have been increased in older adults with sarcopenia, whereas no consensus recommendations on physical exercise for the prevention of sarcopenia have been made due to the existence of multiple contributing variables. Most recently, in 2020, the last published systematic review regarding exercise and sarcopenia showed better physical performance and muscle strength after exercise intervention, but no improvement in muscle mass. Different results from the previous systematic review. Exercise training is also a valuable intervention in cancer cachexia, although most studies have been limited to animal research. Little is known about the exercise benefits of muscle wasting syndrome at present. Previous systematic reviews and meta-analyses have investigated the effects of exercise on either sarcopenic or frail elderly populations; however, the combined meta-analysis approach in elderly adults with sarcopenia and cachexia has not yet been confirmed. Therefore, the aim of this review was to systematically assess the effects of exercise interventions on body composition, muscle strength and functional outcomes in older adults with muscle wasting.

\section{METHODS}

Search strategy: The systematic review was conducted according to the PRISMA standards. A systematic search was performed by a librarian in Six electronic databases, PubMed/Medline, CINAHL, EMBASE, Cochrane Library, and Airiti Library. Moreover, a manual search of the reference lists of relevant reviews and articles included in the systematic review was performed. The following MeSH terms and keywords were used either singularly or in combination: ('older' OR 'aging' OR 'aged' OR 'elderly' OR 'seniors' OR 'geriatric') AND ('sarcopenia' OR 'sarcopenic') OR ('cachexia'[Mesh] OR 'Wasting Syndrome'[Mesh] OR 'cachectic' ) OR ('muscle wasting' OR 'muscle atrophy' OR 'disuse atrophy' OR 'muscle disuse' OR 'myopenia') AND ('exercise' OR 'training' OR 'physical' OR 'progressive resistance training' OR 'resistance exercise' OR 'strength training'). (Online Resource S1). These articles include studies published from 2010 to the present, attributable to SIG's issuing the definition of MWD in 2010. Moreover, a manual search of the reference lists of relevant reviews and articles included in the systematic review was performed. An additional four articles identified through article reference lists were added.

Participant or population: Include the diagnosis of muscle wasting disorder (MND) with an mean or median age $\geq 60$ years, including: muscle wasting, 
according to the EWGSOP and AWGS or Other definitions of sarcopenia, disuse atrophy, cachexia caused by cancer or heart failure, and chronic obstructive pulmonary disease (COPD). In studies where exercise interventions were delivered to patients with cancer and at risk of cachexia, domains for the syndrome including weight loss, nutritional stores and intake, and muscle mass or function were seldom reported. To this end, it was not possible to determine the proportion of patients meeting pre-cachexia or cachexia criteria

Intervention: All types of exercises, resistance exercises, aerobic exercises, vibration exercises, and multi-component regime.

Comparator: usual care or health education.

Study designs to be included: Randomized control trial.

Eligibility criteria: (i) Randomized control trial; (ii) Include the diagnosis of muscle wasting disorder with an mean or median age $\geq 60$ years, including: muscle wasting, according to the EWGSOP and AWGS or Other definitions of sarcopenia, disuse atrophy, cachexia caused by cancer or heart failure, and chronic obstructive pulmonary disease (COPD). In studies where exercise interventions were delivered to patients with cancer and at risk of cachexia, domains for the syndrome including weight loss, nutritional stores and intake, and muscle mass or function were seldom reported. To this end, it was not possible to determine the proportion of patients meeting pre-cachexia or cachexia criteria; (iii) All types of exercises, resistance exercises, aerobic exercises, vibration exercises, and multi-component regime; (iv) Full text articles in Chinese and English; and (v) Outcomes of the studies included at least one of the following data results: muscle mass, muscle strength or physical function.

Information sources: A systematic search was performed by a librarian in Six electronic databases, PubMed/Medline, CINAHL, EMBASE, Cochrane Library, and Airiti Library. Moreover, a manual search of the reference lists of relevant reviews and articles included in the systematic review was performed.

Main outcome(s): A total of 2504 articles were initially identified, and 34 studies were included in the systematic review, including 25 sarcopenia and 9 cachexia (2900 individuals, $38.23 \%$ males, mean age ranged from 60.0 to 88.6 years). The sarcopenia and cachexia were analyzed as subgroups, and the benefits of exercise on sarcopenia are shown, Body composition (ASM [SMD 0.38, $P=0.05]$ and ASM/ height2 [SMD 0.14, $P=0.02]$ ) and Muscle strength (grip strength [SMD 1.73, $P$ < 0.0001]) and physical performance (gait speed [SMD 0.14, $P<0.00001$ ] and the timed up and go test [SMD -1.20, $P<$ $0.00001]$ ) showed significant improvement following exercise treatment. Exercise in the benefit of cachexia, body composition (ASM [SMD 3.38, $P=0.001]$ and physical performance $(400 \mathrm{~m}$ walk [SMD $-36.00, P=$ 0.02] showed significant improvement following exercise treatment, Exercise increased the distance of the 6-minute walking test by $23.56 \mathrm{~m}$ and the Body Weight increased by $3.52 \mathrm{~kg}$, but there was no significant difference, indicating that exercise has potential benefits for patients with cachexia.

Data management: The risk of bias was assessed according to the Cochrane Collaboration's tool for assessing quality and the risk of bias. All steps were performed independently by two investigators, and a third researcher was consulted for suggestions whenever discrepancies existed and decisions were difficult to make. The evaluation included sequence generation, allocation concealment, blinding of participants, personnel or outcome assessors, completeness of outcome data, and selectivity of outcome reporting. Within each domain, an independent judgment by the 2 reviewers, in terms of high, low, or unclear risk of bias, was made. 
Strategy of data synthesis: Descriptive statistics described the extracted data. Meta-analysis was carried out according to the 12 statistic considering low heterogeneity when $12<25 \%$, moderate heterogeneity when $12 \pm 50 \%$ and high heterogeneity when $12>75 \%$. A fixedeffects model was used to estimate the pooled effects if $12<50 \%$, and a randomeffects model was used when $12 \geq 50 \%$. Due to continuous outcome measures, standardised mean differences (SMDs) were calculated as this is more generalizable. If this was not possible, a mean difference was calculated. If the mean difference was lacking, a comparison was made based on the outcome measurements after the intervention. A significance level of $5 \%$ was used for all statistical procedures. Analyses were conducted using Review Manager 5.4.1.

Subgroup analysis: Muscle wasting syndrome includes sarcopenia and cachexia syndromes. In order to avoid the high heterogeneity caused by disease severity, sarcopenia and cachexia are analyzed as subgroups. In addition, due to the multiple types of interventional exercises, the exercise types are divided into resistance exercise, aerobic exercise, whole-body vibration exercise and multicomponent exercise.

Sensitivity analysis: A sensitivity analysis was performed on the following outcomes: muscle strength, muscle mass and physical performance, to investigate the type of exercise on the effect of exercise intervention in older adults with sarcopenia and cachexia. To perform the sensitivity analysis, the primary meta-analysis was repeated, leaving out studies which did not specifically measure the specified factors. Analysis was only carried out when specified factors were measured in two or more studies.

Language: Full text articles in Chinese and English.

Country(ies) involved: Taiwan.
Keywords: Muscle wasting disease; Sarcopenia; Cachexia; Exercise regimen.

Contributions of each author:

Author 1 - Hsiang-Jung Ni.

Email: nini@ms.szmc.edu.tw

Author 2 - Heng-Hsin Tung.

Email: henghsin@ym.edu.tw

Author 3 - Teh-Fu Hsu.

Email: robert.tfhsu@gmail.com

Author 4 - Yu-Chi Chen.

Email: ycchen2@ym.edu.tw

Author 5 - Lok-Hi Chow.

Email: chowlh96@gmail.com

Author 6 - Pei-Fan Mu.

Email: peifan@ym.edu.tw

Author 7 - Ai-Fu Chiou.

Email: afchiou@ym.edu.tw 